\title{
Presence of Wolbachia Endosymbionts in Microfilariae of Wuchereria bancrofti (Spirurida: Onchocercidae) from Different Geographical Regions in India
}

\author{
SL Hoti ${ }^{+}$, A Sridhar, PK Das
}

\author{
Vector Control Research Centre, Indian Council of Medical Research, Indira Nagar, Pondicherry 605006, India
}

In view of the recent discovery of rickettsial endosymbionts, Wolbachia in lymphatic filarial parasites, Wuchereria bancrofti and Brugia malayi and subsequently of their vital role in the survival and development of the latter, antibiotics such as tetracycline are being suggested for the treatment of lymphatic filariasis, by way of eliminating the endosymbiont. But, it is essential to assess their presence in parasites from areas endemic for lymphatic filariasis before such a new control tool is employed. In the present communication, we report the detection of Wolbachia endosymbionts in microfilariae of W. bancrofti parasites collected from geographically distant locations of India, such as Pondicherry (Union Territory), Calicut (Kerala), Jagadalpur (Madhya Pradesh), Thirukoilur (TamilNadu), Chinnanergunam (TamilNadu), Rajahmundry (Andhra Pradesh), and Varanasi (Uttar Pradesh), using Wolbachia specific $16 S$ rDNA polymerase chain reaction.

Key words: Wolbachia - Wuchereria bancrofti - polymerase chain reaction - India

Wolbachia endosymbionts are maternally inherited intracellular $\alpha$-proteobacteria, which infect broad range of invertebrate hosts like insects, mites, isopods, and filarial nematodes (Kellen et al. 1981, Noda 1984, Richardson et al. 1987, Bazzocchi et al. 2000). Wolbachia symbionts have been reported to play important role in the development and pathogenesis of the filarial nematode such as Wuchereria bancrofti, Brugia malayi, and Onchocerca volvulus. Filariasis is mainly characterized by the occurrence of inflammatory pathogenesis and Wolbachia bacteria present in filarial nematodes appear to contribute to this inflammation (Bandi et al. 1998, 1999, Taylor \& Hoerauf 1999, Taylor et al. 2000). The lipopolysaccharide (LPS) of Wolbachia appears to play an important role in elicitation of Toll like receptor-4 (trl-4) signalling which in turn governs the proliferation and regulation of Th1 and Th2 cytokines associated with differential disease manifestations observed in onchocerciasis (André et al. 2002). In view of the im-portance of Wolbachia endosymbionts for the survival, development and pathogenesis of lymphatic filarial parasites in the human host, a new chemotherapeutic strategy targeting the endosymbionts with tetracycline class of compounds is being envisaged for the treatment of filariasis (Taylor \& Hoerauf 1999, Taylor et al. 2000). But, before

Financial support: Filariasis Task Force, Indian Council of Medical Research

${ }^{+}$Corresponding author. Fax: +91-413-2272041. E-mail: slhoti@yahoo.com orvcrc@vsnl.com

Received 24 June 2003

Accepted 16 October 2003 such an approach is implemented on large scale in wider geographical areas, it is necessary to assess the prevalence of the endosymbiont in W. bancrofti from wide geographic locations. At present Wolbachia has been detected only in W. bancrofti microfilariae (mf) from two individuals from Kimuta Island, Papua New Guinea, and Sri Lanka (Taylor et al. 1999, Bazzocchi et al. 2000) and there is no data on their universal prevalence. Hence, a preliminary study was undertaken to screen for the presence of these endosymbionts in $\mathrm{mf}$ of $W$. bancrofti from different geographic regions of India. Five-milliliter blood samples (heparinized) were collected from two or three microfilariaemic individuals residing in each of the five geographically distant locations of India and also from two rural areas near Pondicherry (Figs 1, 2). Microfilariae were separated from blood samples using Millipore membrane $-5.0 \mu \mathrm{M}$ (Millipore, US) following a standard protocol (Sasa 1976) and then purified using Percoll gradient (Chandrasekhar et al. 1984).

DNA was extracted from $\mathrm{mf}$ following the method described by Hoti et al. (2003) which is briefly as follows: the mf pellet was washed in phosphate buffered saline (PBS) and digested in $500 \mu \mathrm{l}$ of lysis buffer [0.1 M Tris-pH 8.5, $0.05 \mathrm{M}$ EDTA, $0.2 \mathrm{M} \mathrm{NaCl}, 1 \%$ sodium dodecyl sulphate (SDS) and proteinase $\mathrm{K} 2 \mathrm{mg} / \mathrm{ml}$. The lysate was subjected to phenol: chloroform: isoamylalcohol (25:24:1) extraction followed by ethanol precipitation and pellet was stored in $20 \mu \mathrm{l}$ of $6 \mathrm{mM}$ Tris, $6 \mathrm{mM} \mathrm{NaCl}$, 0.2 mM EDTA; $\mathrm{pH} 7.0$.

Wolbachia specific 16S rDNA primers, forward primer 27f (5'-GAGATTTGATCCTGGCTCAG-3' and reverse primer 1495r (5'-CTACGGCTACCTTGTTACGA-3') were used to amplify Wolbachia ribosomal genes from the genomic DNA of mf (Taylor et al. 1999). The genomic positions of forward primer and reverse primer in $16 \mathrm{~S}$ 


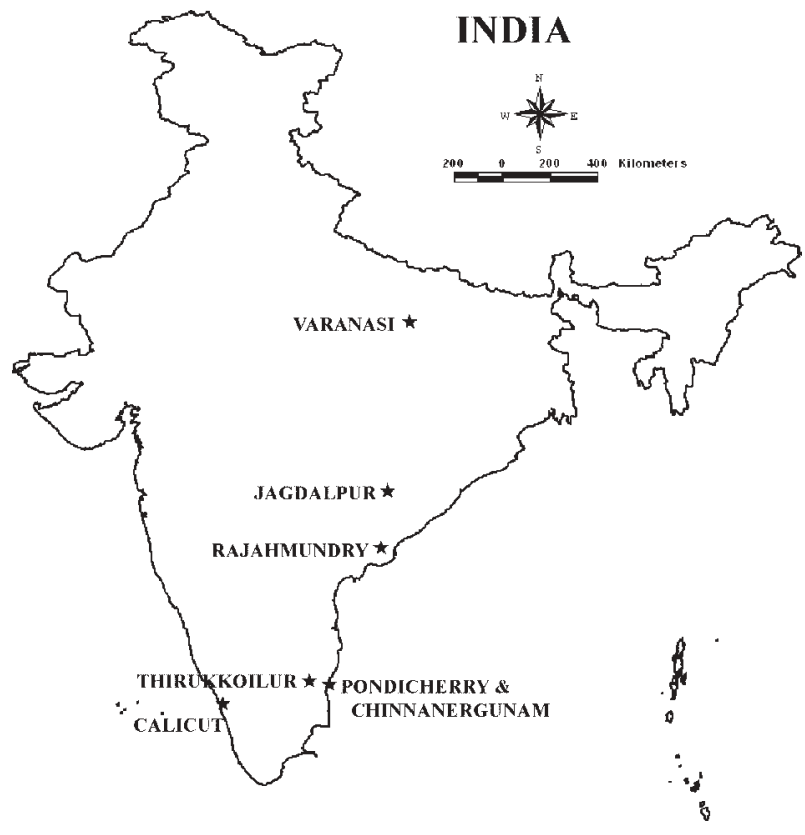

Fig. 1: map showing different geographic locations of India from where microfilariae of Wuchereria bancrofti were collected. ribosomal gene lie at nucleotide position 27 to 46 and 1495 to 1515 , respectively. The polymerase chain reaction (PCR) mixture consisted of dNTP - $20 \mathrm{mM}$ of each nucleotide (Finnzymes, Finland); $\mathrm{MgCl}_{2}-2.5 \mathrm{mM}$ (Finnzymes); Forward and Reverse Primer (Synthesized from Metabion, Germany) - 10 pmol each, 10X Dynazyme buffer, Dynazyme II Taq polymerase - 3 units (Finnzymes, Finland), template mf DNA $10 \mathrm{ng}$ and distilled water (Sigma, US). B. malayi mf DNA known to harbor Wolbachia endosymbiont ribosomal gene was used as positive control and distilled water instead of template DNA was used as negative control for PCR. Amplification was run on a Master Cycler Gradient (Eppendorf). The PCR programme consisted of an initial denaturation at $96^{\circ} \mathrm{C}$ for $4 \mathrm{~min}$ followed by 35 cycles of denaturation at $94^{\circ} \mathrm{C}$ for $1 \mathrm{~min}$, annealing at $56^{\circ} \mathrm{C}$ for $1 \mathrm{~min}$ and extension at $72^{\circ} \mathrm{C}$ for $2 \mathrm{~min}$ with a final extension step of $72^{\circ} \mathrm{C}$ for 7 min. The amplified products were detected by running a $1.5 \%$ agarose gel with Lambda DNA/Hind III and Phi X 174 DNA/Hae III Mix molecular weight marker (NEB, US). The gels were stained in ethidium bromide, observed under UV transilluminator and then photographed (Fig. 2a, b).

Results of the study (Fig. 2a, b) showed that the PCR amplification using the eubacterial primers yielded an amplicon of $1400 \mathrm{bp}$ which corresponds to $16 \mathrm{~S}$ rDNA gene
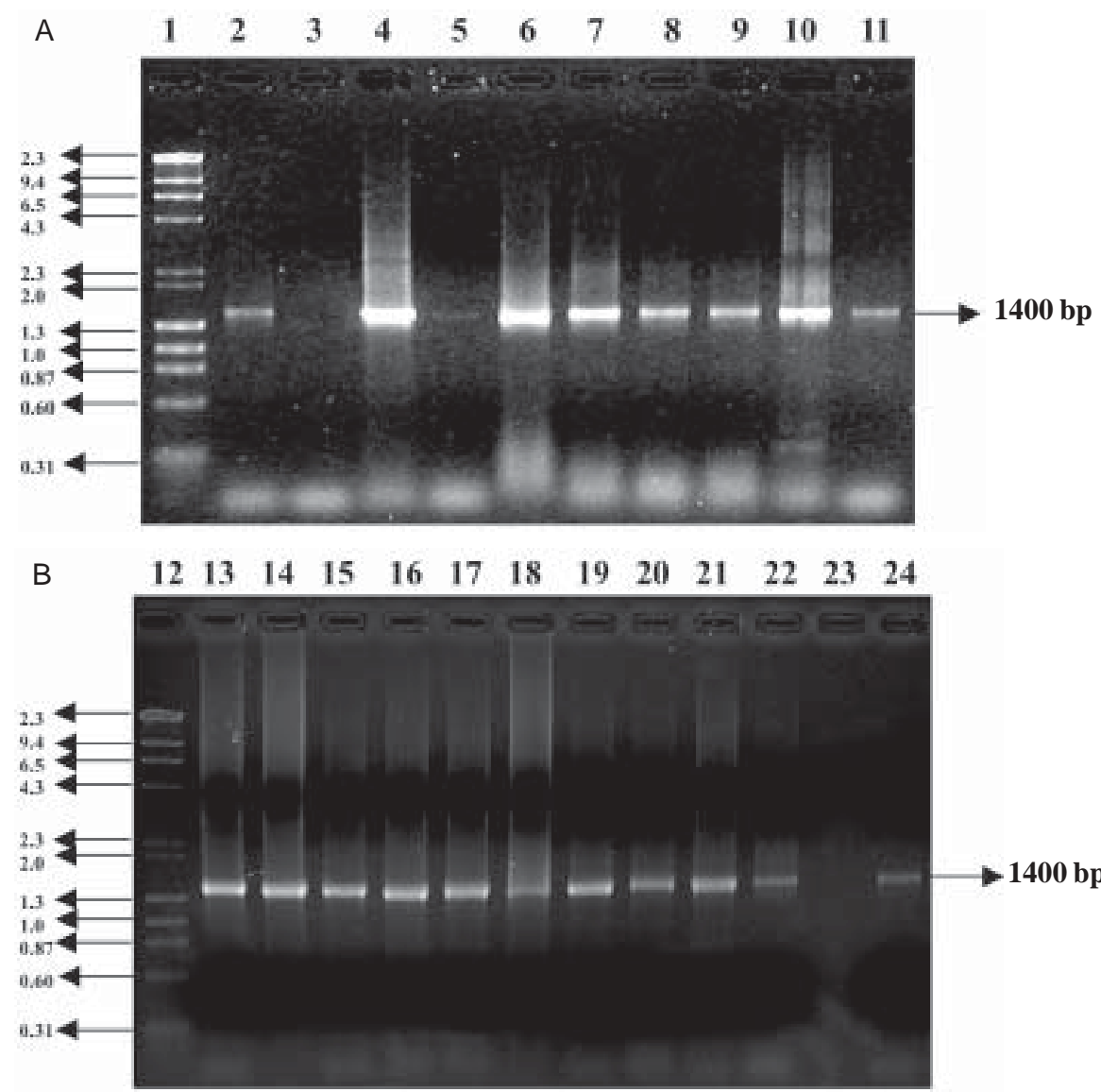

Fig. 2: amplification of 16s rDNA of Wolbachia from Wuchereria bancrofti microfilariae collected from different geographic regions of India. A: lanes - 1: mol. wt marker; 2: positive control; 3: negative control; 4-6: Pondicherry isolates; 7-9: Calicut isolates; 10-11: Varanasi isolates; B: lanes - 12: mol. wt marker; 13-15: Jagadalpur isolates; 16-17: Thirukoilur isolates; 18-20: Chinnanergunam isolates; 21-22: Rajahmundry isolates; 23: negative control; 24: positive control 
of Wolbachia, from W. bancrofti mf isolates collected from different geographic locations of India viz., Pondicherry (Union Territory), Calicut (Kerala State), Jagadalpur (Chattisgarh State), Thirukoilur (TamilNadu), Chinnanergunam (TamilNadu), Rajahmundry (Andhra Pradesh), and Varanasi (Uttar Pradesh) spanning about $2000 \mathrm{~km}$. This is similar to what has been reported for Wolbachia endosymbionts of filarial nematodes by other workers (Taylor et al. 1999). Thus, the preliminary study showed that Wolbachia endosymbionts were present in populations of $W$. bancrofti originating from wider geographic areas of India.

\section{ACKNOWLEDEGMENTS}

To Dr K Balaraman, Deputy Director (Senior Grade), Vector Control Research Centre, Pondicherry for critically reviewing the manuscript. To Dr RC Sharma, Head of Helminthology Division and the staff of National Institute of Communicable Diseases, Delhi, India for their help in the collection of blood samples from microfilariae carriers

\section{REFERENCES}

André A, Blackwell NM, Hall LR, Hoerauf A, Brattig NW, Volkmann L, Taylor MJ, Ford L, Hise AG, Lass JH, Diaconu E, Pearlman E 2002. The role of endosymbiotic Wolbachia bacteria in the pathogenesis of river blindness. Science 295: 1892-1895.

Bandi C, Anderson TJC, Genchi C, Blaxter ML 1998. Phylogeny of Wolbachia-like bacteria in filarial nematodes. Proc $R$ Soc London Ser B 265: 2407-2413.

Bandi C, Slatko B, O’Neill SL 1999. Wolbachia genomes and the many faces of symbiosis. Parasitol Today 15: 428-429.

Bazzocchi C, Jamnongluk W, O'Neill SL, Anderson TJ, Genchi C, Bandi C 2000. wsp gene sequences from the Wolbachia of filarial nematodes. Curr Microbiol 41: 96-100.

Chandrasekar R, Rao UR, Rajasekhariah GR, Subrahmanyam D 1984. Isolation of microfilariae from blood on iso-osmotic gradients. Indian J Med Res 79: 497-501.

Hoti SL, Subramaniyan K, Das PK 2003. Detection of codon for aminoacid 200 in isotype $1 \beta$-tubulin gene of Wuchereria bancrofti isolates, implicated in resistance to benzimidazoles in other nematodes. Acta Trop 88: 77-81.

Kellen WR, Hoffmann DF 1981. Wolbachia sp. a symbiont of the almond moth, Ephestia cautella: ultrastructure and influence on host fertility. J Invert Pathol 37: 273-283.

Noda H 1984. Cytoplasmic incompatibility in a plant ricehopper. $J$ Hered 75: 345-348.

Richardson PM, Holmes WP, Saul GB II 1987. The effect of tetracycline on reciprocal cross incompatibility in Mormoniella [=Nasonia] vitripennis. J Invert Pathol 50: 176-183.

Sasa M 1976. Human Filariasis - A Global Survey of Epidemiology and Control, University of Tokyo press, Tokyo, $334 \mathrm{pp}$.

Taylor MJ, Hoerauf A 1999. Wolbachia bacteria of filarial nematodes. Parasitol Today 15: 437-442.

Taylor MJ, Bilo K, Cross HF, Archer JP, Underwood AP 1999. 16S rDNA phylogeny and ultrastructural characterization of Wolbachia intracellular bacteria of the filarial nematodes Brugia malayi, Brugia pahangi, and Wuchereria bancrofti. Exp Parasitol 91: 356-361.

Taylor MJ, Bandi C, Hoerauf AM, Lazdins 2000. Wolbachia bacteria of filarial nematodes: a target for control? Parasitol Today 16: 179-180. 